\title{
The Multiwavelength AGN Population and the X-ray Background
}

\author{
Ezequiel Treister ${ }^{1}$, Claudia M. Urry ${ }^{2}$, Kevin Schawinski ${ }^{3}$, \\ Brooke D. Simmons ${ }^{4}$, Priyamvada Natarajan ${ }^{5}$ and Marta Volonteri ${ }^{6}$ \\ ${ }^{1}$ Universidad de Concepción, Departamento de Astronomía, Casilla 160-C, Concepción, Chile \\ email: etreiste@astro-udec.cl \\ ${ }^{2}$ YCAA \& Dept. of Physics, Yale University, P.O. Box 208121, New Haven, CT 06520 \\ ${ }^{3}$ Institute for Astronomy, Department of Physics, ETH Zurich, \\ Wolfgang-Pauli-Strasse 16, CH-8093 Zurich, Switzerland \\ ${ }^{4}$ Department of Physics, University of Oxford, Keble Road, Oxford OX1 3RH, UK. \\ ${ }^{5}$ YCAA, Dept. of Astronomy \& Dept. of Physics, Yale University, \\ P.O. Box 208121, New Haven, CT 06520 \\ ${ }^{6}$ Institut d' Astrophysique de Paris, 98bis Bd. Arago, 75014 Paris, France
}

\begin{abstract}
In order to fully understand galaxy formation we need to know when in the cosmic history are supermassive black holes (SMBHs) growing more intensively, in what type of galaxies this growth is happening and what fraction of these sources are invisible at most wavelengths due to obscuration. Active Galactic Nuclei (AGN) population synthesis models that can explain the spectral shape and intensity of the cosmic X-ray background (CXRB) indicate that most of the SMBH growth occurs in moderate-luminosity $\left(\mathrm{L}_{X} \sim 10^{44} \mathrm{erg} / \mathrm{s}\right.$ ) sources (Seyfert-type AGN), at $z \sim 0.5-1$ and in heavily obscured but Compton-thin, $N_{H} \sim 10^{23} \mathrm{~cm}^{-2}$, systems. However, this is not the complete history, as a large fraction of black hole growth does not emit significantly in X-rays either due to obscuration, intrinsic low luminosities or large distances. The integrated intensity at high energies indicates that a significant fraction of the total black hole growth, $22 \%$, occurs in heavily-obscured systems that are not individually detected in even the deepest $\mathrm{X}$-ray observations. We further investigate the AGN triggering mechanism as a function of bolometric luminosity, finding evidence for a strong connection between significant black hole growth events and major galaxy mergers from $z \sim 0$ to $z \sim 3$, while less spectacular but longer accretion episodes are most likely due to other (stochastic) processes. AGN activity triggered by major galaxies is responsible for $\sim 60 \%$ of the total black hole growth. Finally, we constrain the total accreted mass density in supermassive black holes at $z>6$, inferred via the upper limit derived from the integrated X-ray emission from a sample of photometrically selected galaxy candidates. We estimate an accreted mass density $<1000 \mathrm{M}_{\odot} \mathrm{Mpc}^{-3}$ at $z \sim 6$, significantly lower than the previous predictions from some existing models of early black hole growth and earlier prior observations.
\end{abstract}

Keywords. galaxies: active, galaxies: high-redshift, quasars: general, X-rays: diffuse background

\section{Introduction}

It is now clear that the formation of the supermassive black holes (SMBHs; mass greater than a million solar masses) that can be now found in the centers of most massive galaxies (e.g., Kormendy \& Richstone 1995) is directly connected to the evolution of their host galaxies (Ferrarese \& Merritt 2000; Gebhardt et al. 2000). However, the details of this connection are still not well understood. In order to fully understand galaxy formation we need to know when in the cosmic history are black holes (BHs) growing more intensively, 

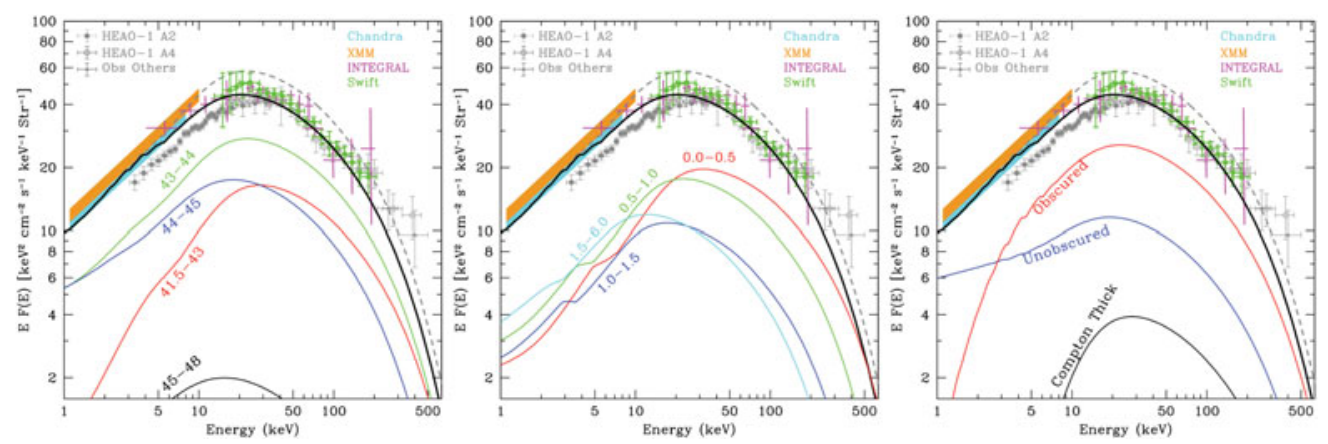

Figure 1. Observed spectrum of the extragalactic XRB from HEAO-1 (Gruber et al. 1999), Chandra (Hickox \& Markevitch 2006), XMM (De Luca \& Molendi 2004), INTEGRAL (Churazov et al. 2007) and Swift (Ajello et al. 2008) data. In each panel the thick black solid line shows the population synthesis model for the XRB spectrum of Treister et al. (2009a). Left panel: Contribution to the XRB as a function of intrinsic X-ray luminosity for sources with $L_{X}<10^{43} \mathrm{erg} / \mathrm{s}$ (red line), $10^{43}<L_{X}<10^{44}$ (green line), $10^{44}<L_{X}<10^{45}$ (blue line) and $L_{X}>10^{45} \mathrm{erg} / \mathrm{s}$ (thin black line). Center panel: Contribution to the XRB from sources at $z<0.5$ (red line), $0.5<z<1$ (green line), $1<z<1.5$ (blue line) and $z>1.5$ (cyan line). Right panel: Contribution to the XRB from unobscured (blue line), obscured but Compton-thin (red line) and Compton-thick sources (thin black line).

in what type of galaxies this growth is happening and what fraction of these sources are invisible at most wavelengths due to obscuration.

The spectral shape and intensity of the extragalactic Cosmic X-ray background (CXRB) can be used as an integral constraint for the statistical properties of the Active Galactic Nuclei (AGN) population, and thus the amount of BH growth occurring in these systems and their evolution. This is because most recent deep surveys show that $\sim 90 \%$ of the observed 2-8 keV CXRB radiation can be attributed to resolved AGN (e.g., Hickox \& Markevitch 2006).

\section{AGN and the Cosmic X-ray Background}

As can be seen in Figure 1, the CXRB shows that most of the BH accretion that can be traced in X-rays can be attributed to obscured but Compton-thin, moderate-luminosity systems $\left(L_{X} \sim 10^{44} \mathrm{erg} / \mathrm{s}\right.$, corresponding to $\left.L_{b o l} \sim 10^{45} \mathrm{erg} / \mathrm{s}\right)$ at relatively low redshifts $(z<1)$. While the results presented here are based on the Treister et al. (2009a) CXRB model, similar results are obtained if other recent computations are considered (e.g., Treister \& Urry 2005; Ballantyne et al. 2006; Gilli et al. 2007).

While these conclusions are certainly interesting, it is worth remembering that the XRB provides only an integral flux constraint, and therefore less obscured sources at lower redshifts carry more weight. It is certainly possible that a large fraction of the total $\mathrm{BH}$ accretion occurs in heavily obscured, even Compton-thick, AGN at high redshifts. Indeed, while heavily obscured AGN contribute only $\sim 5 \%$ of the total CXRB emission (Treister et al. 2009a) they can account for up to $\sim 30 \%$ of the total BH mass density at $z=0$ (Treister et al. 2010). As can be seen in Figure 2, the sources contributing most of the XRB represent only a small fraction of the total AGN population, which in turn corresponds to $\sim 50-60 \%$ of the total $\mathrm{BH}$ growth. In addition, most X-ray surveys, and hence the CXRB, are not very sensitive to low luminosity sources, which can however be detected in luminous infrared galaxies. For example, Treister et al. (2010) reported that the fraction of moderate-luminosity Compton-thick sources in a sample of galaxies with $L_{I R}>5 \times 10^{10} L_{\odot}$, relative to the X-ray detected AGN in the CDF-S, is $\sim 25 \%$. Similarly, 


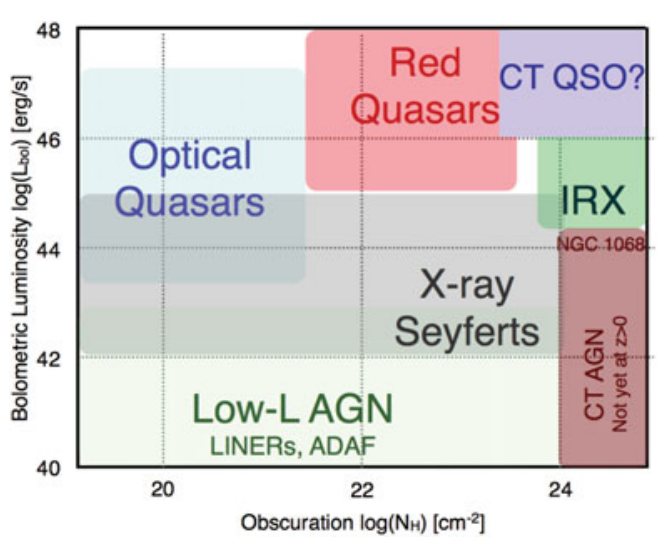

Figure 2. Different AGN families separated as a function of their intrinsic bolometric luminosities in the $\mathrm{y}$ axis and the amount of obscuration in the line of sight in the $\mathrm{x}$ axis. As shown before, most of the X-ray background come sources with $L_{b o l}<10^{45} \mathrm{erg} / \mathrm{s}$ and $N_{H}<10^{24} \mathrm{~cm}^{-2}$. Obscured high-luminosity sources can contribute up to $30 \%$ of the total $\mathrm{BH}$ growth (Treister et al. 2010), while low luminosity sources, typically missed in X-ray surveys might represent an additional $\sim 10 \%$ contribution to the total $\mathrm{BH}$ growth. Those sources can be detected as luminous infrared galaxies (Treister et al. 2010).

while high redshift sources do not contribute significantly to the CXRB, they are critical in our understanding of how the first SMBHs formed, as discussed in section 4.

\section{What triggers AGN activity?}

While it is clear now that most galaxies contain a supermassive $\mathrm{BH}$ in their center, in only relatively few cases is this black hole actively growing. This indicates that $\mathrm{BH}$ growth is most likely episodic, with each luminous event lasting $\sim 10^{7}-10^{8}$ years (Di Matteo et al. 2005). Hence, an obvious question is what triggers these BH growth episodes?

Major galaxy mergers provide a good explanation, since as simulations show, they are very efficient in driving gas to the galaxy center (Barnes \& Hernquist 1991), where it can be used as fuel for both intense circumnuclear star formation and BH growth. Indeed, a clear link between quasar activity and galaxy mergers has been seen in intensely starforming galaxies like Ultra-luminous infrared galaxies (ULIRGs) and in some luminous quasars (e.g., Sanders et al. 1988). In contrast, many AGN are clearly not in mergers or especially rich environments (De Robertis et al. 1998) and the prevalence of disks in most AGN host galaxies rules out frequent major mergers (Schawinski et al. 2011a, 2012; Simmons et al. 2012). Instead, minor interactions (Moore et al. 1996), instabilities driven by galaxy bars (Kormendy \& Kennicutt 2004) and other internal galaxy processes might be responsible for these lower activity levels. Understanding the role of mergers is further complicated by the difficulty of detecting merger signatures at high redshifts.

In order to reconcile these potentially contradictory observations it has been suggested that the AGN triggering mechanism is a function of luminosity and/or redshift (Finn et al. 2001, and others). More recently, Hopkins \& Hernquist (2009) used five indirect tests to conclude that the triggering mechanism is strongly luminosity-dependent and more weakly redshift-dependent, so that only the most luminous sources, which are preferentially found at $z>2$, are triggered by major mergers. Thanks to results from large AGN surveys, which now include heavily-obscured IR-selected sources, and recent deep high-resolution observations carried out with the Hubble WFC3 detector, it is now possible to obtain reliable morphological information even for high- $z$, low luminosity sources. 


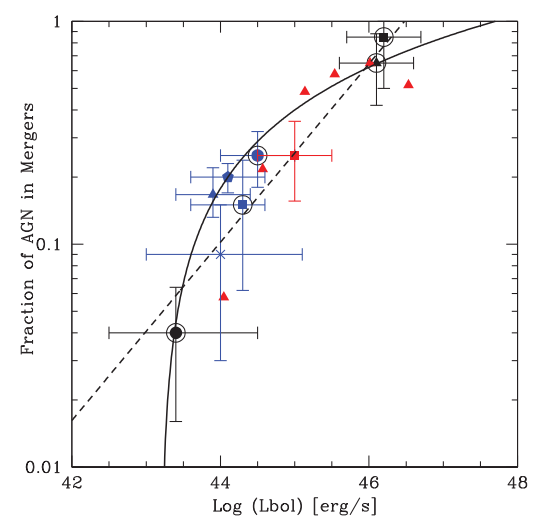

Figure 3. Fraction of AGN showing mergers as a function of the AGN bolometric luminosity, from the work of Treister et al. (2012). Colors indicate AGN selection method (red: infrared, blue: X-rays, black: optical). Encircled symbols show samples at $z<1$. Solid line shows a fit to the data assuming a linear dependence of the fraction on $\log \left(L_{b o l}\right)$, while the dashed line assumes a power-law dependence.

To measure the fraction of AGN hosted by a galaxy undergoing a major merger as a function of luminosity and redshift, Treister et al. (2012) compiled information from AGN samples selected from X-ray, infrared and spectroscopic surveys. They studied data from 10 independent surveys, which include 874 AGN, spanning a wide range in luminosities, $3 \times 10^{42}<\mathrm{L}_{\text {bol }}\left(\mathrm{erg} \mathrm{s}^{-1}\right)<5 \times 10^{46}$, and redshift, $0<z<3$. The goal of that work was to determine the physical mechanism(s) that provoked the AGN activity identified in these surveys. Only visual morphological classifications were used, as they are the most reliable option to determine if a galaxy is experiencing a major merger (Darg et al. 2010). The fraction of AGN linked to galaxy mergers in these samples has been computed by dividing the number of AGN in which the host galaxy has been classified as an ongoing merger or as having major disturbances by the total number of AGN. Figure 3 shows the fraction of AGN showing mergers as a function of bolometric luminosity, which increases rapidly, from $\sim 4 \%$ at $10^{43} \mathrm{erg} \mathrm{s}^{-1}$ to $\sim 90 \%$ at $10^{46} \mathrm{erg} \mathrm{s}^{-1}$.

As reported by Treister et al. (2012), about half of the BH growth happens in quasarlike AGN following a major merger, while the other half occurs in secularly-fueled lowerluminosity AGN. At relatively high redshifts, $z \gtrsim 2$, there is $\sim 60 \%$ more BH growth in merger-triggered AGN than in those growing via secular processes. At lower redshifts, there are relatively fewer galaxy mergers and so secular processes become slightly more important. In terms of numbers, the population is strongly dominated by secularlytriggered AGN. Indeed, Treister et al. (2012) concluded that $~ 90 \%$ of the AGN at all redshifts are associated with secular processes. This explains the conclusions of previous studies, mostly based on X-ray surveys of moderate luminosity AGN, that found that normal disk-dominated galaxies constitute the majority of the AGN host galaxies. So, we conclude that while most AGN are triggered by secular processes, a slight majority of the BH growth, particularly at high redshifts, can be attributed to intense accretion episodes linked to major galaxy mergers.

\section{The first SMBH seeds}

One of the most challenging problems in astronomy today is understanding how and when the first SMBHs in the Universe formed. We are now in a position to examine this issue with adequate data combining sources selected as part of the Hubble Ultra Deep 

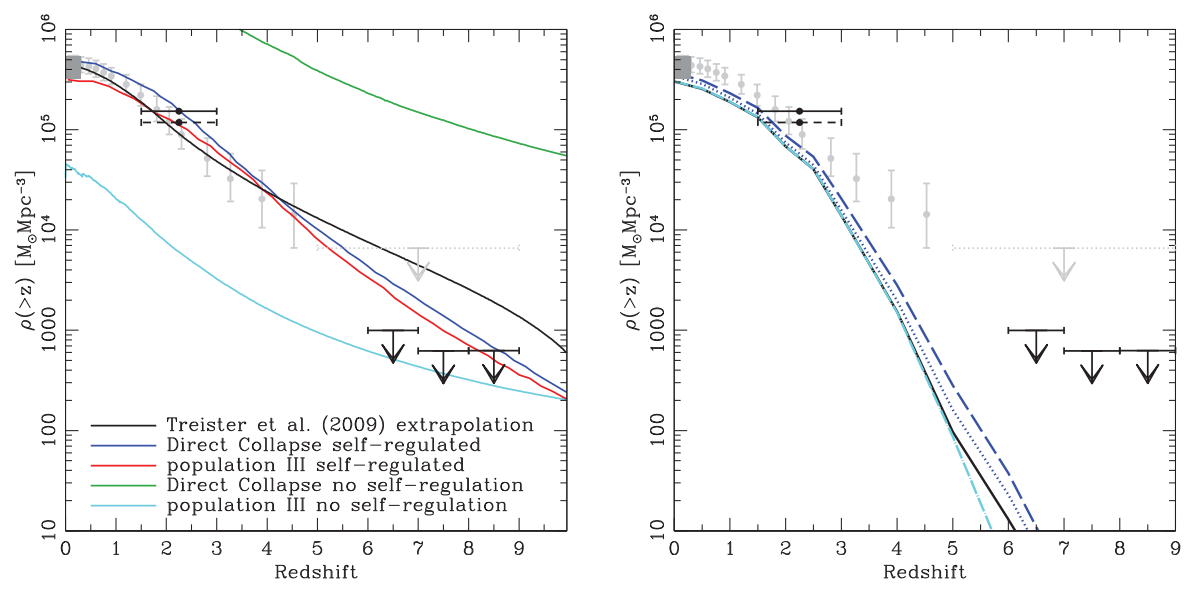

Figure 4. Accreted BH mass density as a function of redshift. In both panels the gray rectangles show the range of values allowed by observations of $z \simeq 0$ galaxies (Shankar et al. 2009), while the filled circles show the observations of $\mathrm{BH}$ accreted mass density traced by AGN activity in the $0<z<5$ range compiled by Hopkins et al. (2007) and measured by Treister et al. (2009b) at $z \sim 2$. At higher redshifts, we show the CXRB integral constraint derived by Salvaterra et al. (2012, upper limit with dotted error bars) and the upper limits at $z=6-9$ obtained by Treister et al. (2013). Left Panel: Expectations from the models of Volonteri (2010). The red and blue lines show the predicted BH mass density if self-regulation, is incorporated, for Pop III and direct collapse BH seeds respectively. The cyan and green lines show the results from these models if no regulation is assumed.Right panel: Expectations obtained from the models of Bonoli et al. (2014). The dot-dashed cyan line only considers light (Pop III) BH seeds, while the solid black line and the blue dotted line include both Pop III and direct collapse seeds. The dashed blue line also considers both seed types, but in this case massive seeds can also form in 1:10 mergers. For these models, marginal agreement with observations is obtained at $z<3$, while they are at the same time consistent with our upper limits at $z>6$.

Field (HUDF) and the Cosmic Assembly Near-IR Deep Extragalactic Legacy Survey (CANDELS). In Treister et al. (2013), we presented the X-ray properties of samples of $z \sim 6,7$ and 8 galaxy candidates in these fields. None of these galaxies are detected in Xray, either individually or collectively via stacking. This non-detection via the consequent upper limit on the accreted mass density of $<1000 \mathrm{M}_{\odot} \mathrm{Mpc}^{-3}$, offers the most stringent constraints on $\mathrm{BH}$ growth in the early Universe. This is particularly surprising, as our X-ray stacking observations are sensitive enough to detect even moderate amounts of accretion in relatively small BHs. Furthermore, such low accretion levels contradict the predictions of several BH formation and evolution models, as shown in Figure 4.

Explaining these results requires that these high-redshift dropout galaxies, which are now routinely found and studied by HST and large ground-based telescopes, (A) do not contain SMBHs, or (B) if they contain BHs, then these are not growing, or (C) the $\mathrm{BH}$ growth if occurring is heavily obscured and/or not radiating efficiently in X-rays. If $\mathrm{BHs}$ are present in these galaxies, we return to the question of why they are not accreting, in particular since they appear to have significant amounts of gas, given their high specific star formation rates. If they do not contain $\mathrm{BHs}$, then our results have new implications for BH seed formation mechanisms; namely, that normal star-forming galaxies at $6<z<8$ are not forming/growing $\mathrm{BH}$ seeds at their centers. This may indicate that seed formation and growth in the general galaxy population can be delayed in some cases until much lower redshifts, as suggested by Volonteri \& Begelman (2010) and more recently by Bonoli et al. (2014). A particularly remarkable example of such systems was discovered and reported by Schawinski et al. (2011b) at $z=1.35$. Our results strongly 


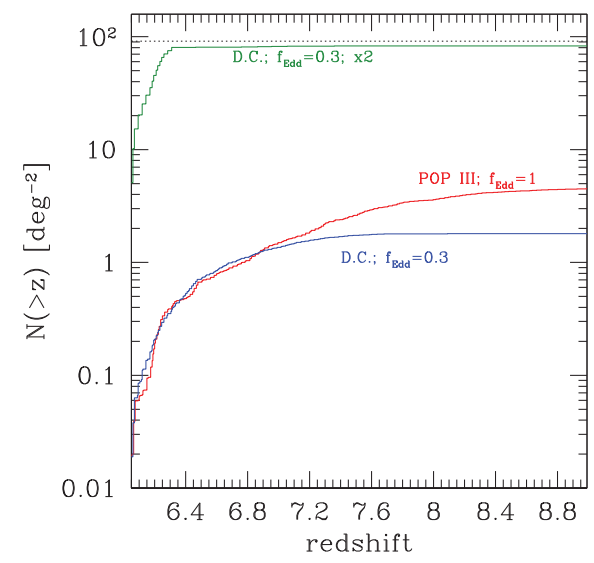

Figure 5. Cumulative number of sources as a function of redshift for individual X-ray detections. This calculation assumes the X-ray flux limit of the 4 Msec CDF-S Chandra observations. The horizontal dotted line shows the number density required to individually detect one source at $z>7$ in the CDF-S. Solid lines represent the expected source densities for different BH seed formation scenarios, based on the models of Volonteri (2010), as labelled in the figure (Pop III, $f_{\mathrm{Edd}}=1$; D. C., $f_{\mathrm{Edd}}=0.3$; D. C., $f_{\mathrm{Edd}}=0.3, \times 2$ ). Note: model Pop III, $f_{\mathrm{Edd}}=1, \times 2$ has no detectable source. To distinguish between these models for early $\mathrm{BH}$ formation will require a deep multiwavelength survey covering at least $\sim 1 \mathrm{deg}^{2}$.

suggest that the individual sites where the bulk of the stars form at $6<z<8$ may not be the sites that harbor the most massive BHs.

There are two main scenarios for the formation of the first SMBHs. The first one postulates that $\mathrm{BH}$ seeds result from the remnants of the first stars, the so-called population III generation stars (e.g., Abel et al. 2000; Madau \& Rees 2001; Bromm et al. 2002). The second possibility is a heuristic picture wherein early BH seeds could form via direct gravitational collapse of gas-rich pre-galactic disks, leading to significantly more massive seed masses with $M_{\text {seeds }} \sim 10^{5} M_{\odot}$ (Loeb \& Rasio 1994; Bromm \& Loeb 2003; Begelman et al. 2006; Lodato \& Natarajan 2006). As argued by Treister et al. (2011); Treister et al. (2013), separating these two scenarios requires to detect individual growing SMBHs, in the early stages of their growth, i.e. significantly before the quasar phase, independent of the amount of obscuration in the line of sight. Therefore, such as study requires a deep X-ray survey, reaching flux limits on the order of $10^{-17} \mathrm{erg} \mathrm{cm}^{-2} \mathrm{~s}^{-1}$, extending over at least a $1 \mathrm{deg}^{2}$ area, but ideally covering $\sim 5 \mathrm{deg}^{2}$ in order to obtain a significant number of these sources. Such study is unrealistic using currently-available facilities such as Chandra or $X M M-N e w t o n$. Indeed, it will require at least $50 \mathrm{Msec}$ (about 1.5 years on-target or $\sim 2$ years including overheads) using Chandra. Therefore, it is our opinion that a dedicated mission is required to answer this scientific question, one that at the same time is as sensitive as Chandra but with a field of view $\sim 10 \times$ larger. Luckily, thanks to the advances in X-ray optics and detector technology, and the fact that only moderate spatial resolution, $\sim 5^{\prime \prime}$ is required, such a telescope can be built as a moderate-size explorer mission, easily within reach in the next 10 years.

\section{Acknowledgements}

We thank the organizers for a very interesting and stimulating conference. In particular, ET would like to thank them for the opportunity to give an invited talk. Support for the work of ET is provided by the Center of Excellence in Astrophysics and Associated 
Technologies (PFB 06), by the FONDECYT grant 1120061 and by the Anillo project ACT1101.

\section{References}

Abel, T., Bryan, G. L., \& Norman, M. L. 2000, ApJ, 540, 39

Ajello, M. et al. 2008, ApJ, 689, 666

Ballantyne, D. R., Everett, J. E., \& Murray, N. 2006, ApJ, 639, 740

Barnes, J. E. \& Hernquist, L. E. 1991, ApJL, 370, L65

Begelman, M. C., Volonteri, M., \& Rees, M. J. 2006, MNRAS, 370, 289

Bonoli, S., Mayer, L., \& Callegari, S. 2014, MNRAS, 437, 1576

Bromm, V., Coppi, P. S., \& Larson, R. B. 2002, ApJ, 564, 23

Bromm, V. \& Loeb, A. 2003, ApJ, 596, 34

Churazov, E. et al. 2007, A\&A A, 467, 529

Darg, D. W., Kaviraj, S., Lintott, C. J., et al. 2010, MNRAS, 401, 1552

De Luca, A. \& Molendi, S. 2004, A\& A, 419, 837

De Robertis, M. M., Yee, H. K. C., \& Hayhoe, K. 1998, ApJ, 496, 93

Di Matteo, T., Springel, V., \& Hernquist, L. 2005, Nature, 433, 604

Ferrarese, L. \& Merritt, D. 2000, ApJL, 539, L9

Finn, R. A., Impey, C. D., \& Hooper, E. J. 2001, ApJ, 557, 578

Gebhardt, K. et al. 2000, ApJL, 539, L13

Gilli, R., Comastri, A., \& Hasinger, G. 2007, A\&A, 463, 79

Gruber, D. E., Matteson, J. L., Peterson, L. E., \& Jung, G. V. 1999, ApJ, 520, 124

Hickox, R. C. \& Markevitch, M. 2006, ApJ, 645, 95

Hopkins, P. F., Richards, G. T., \& Hernquist, L. 2007, ApJ, 654, 731

Hopkins, P. F. \& Hernquist, L. 2009, ApJ, 694, 599

Kormendy, J. \& Richstone, D. 1995, ARAEAA, 33, 581

Kormendy, J. \& Kennicutt, Jr., R. C. 2004, ARA\&A, 42, 603

Lodato, G. \& Natarajan, P. 2006, MNRAS, 371, 1813

Loeb, A. \& Rasio, F. A. 1994, ApJ, 432, 52

Madau, P. \& Rees, M. J. 2001, ApJL, 551, L27

Moore, B., Katz, N., Lake, G., Dressler, A., \& Oemler, A. 1996, Nature, 379, 613

Salvaterra, R., Haardt, F., Volonteri, M., \& Moretti, A. 2012, A $\& A$, 545, L6

Sanders, D. B., Soifer, B. T., Elias, J. H., et al. 1988, ApJ, 325, 74

Schawinski, K., Treister, E., Urry, C. M., et al. 2011, ApJL, 727, L31

Schawinski, K., Urry, M., Treister, E., Simmons, B., Natarajan, P., \& Glikman, E. 2011, ApJL, 743, L37

Schawinski, K., Simmons, B. D., Urry, C. M., Treister, E., \& Glikman, E. 2012, MNRAS, 425, L61

Shankar, F., Weinberg, D. H., \& Miralda-Escudé, J. 2009, ApJ, 690, 20

Simmons, B. D., Urry, C. M., Schawinski, K., Cardamone, C., \& Glikman, E. 2012, ApJ, 761, 75

Treister, E. \& Urry, C. M. 2005, ApJ, 630, 115

Treister, E., Urry, C. M., \& Virani, S. 2009a, ApJ, 696, 110

Treister, E. et al. 2009b, ApJ, 706, 535

Treister, E., Natarajan, P., Sanders, D. B., et al. 2010, Science, 328, 600

Treister, E., et al. 2010, ApJL, 722, L238

Treister, E., Schawinski, K., Volonteri, M., Natarajan, P., \& Gawiser, E. 2011, Nature, 474, 356

Treister, E., Schawinski, K., Urry, C. M., \& Simmons, B. D. 2012, ApJL, 758, L39

Treister, E., Schawinski, K., Volonteri, M., \& Natarajan, P. 2013, ApJ, 778, 130

Volonteri, M. 2010, A\&SARv, 18, 279

Volonteri, M. \& Begelman, M. C. 2010, MNRAS, 409, 1022 